FORMATION Formation emploi

Revue française de sciences sociales

128 | octobre-décembre 2014

Pêle-Mêle

\title{
Les inégalités dans la formation des élus locaux
}

The inequalities in the training of local elected representatives

Ungleichheiten bei der Ausbildung von Kommunalpolitikern

Las desigualdades en la formación de los políticos locales

\section{Maud Navarre}

\section{OpenEdition}

Journals

Édition électronique

URL : http://journals.openedition.org/formationemploi/4310

DOI : 10.4000/formationemploi.4310

ISSN : 2107-0946

Éditeur

La Documentation française

Édition imprimée

Date de publication : 31 décembre 2014

Pagination : 65-79

ISSN : 0759-6340

Référence électronique

Maud Navarre, "Les inégalités dans la formation des élus locaux», Formation emploi [En ligne], 128| octobre-décembre 2014, mis en ligne le 14 janvier 2015, consulté le 30 octobre 2020. URL : http:// journals.openedition.org/formationemploi/4310 ; DOI : https://doi.org/10.4000/formationemploi.4310

(C) Tous droits réservés 


\section{Les inégalités dans la formation des élus locaux}

Maud NavarRe

Docteure en sociologie, attachée temporaire d'enseignement et de recherche (ATER) en sciences de l'éducation, à l'université de Bourgogne, Centre Georges Chevrier (UMR CNRS

Résumé

Les inégalités dans la formation des élus locaux

L'article étudie l'inégal usage des différents modes de formation par les élus locaux. Ces derniers mobilisent peu les dispositifs prévus par la loi, tels que les stages, préférant un apprentissage sur le tas. Ces deux modes de formation s'avèrent inégalement accessibles et efficaces selon le sexe et le niveau de qualification. Les inégalités sont d'autant plus prégnantes que l'usage des dispositifs destinés à la formation des élus est faible. Cette caractéristique constitue la spécificité du milieu politique par rapport au milieu professionnel.

\section{Mots clés :}

Formation sur le tas, métier de la politique, division sexuelle de la formation, récit de vie, stage de formation

Abstract

The inequalities in the training of local elected representatives

The article studies the uneven use of various modes of training by local elected representatives. They employ statutory devices as internships less than on-the-job training. These two modes of training turn out unevenly accessible and effective according to sex and qualification. Disparities are all the stronger as the use of devices intended for the training of the elected representatives is low. This is the specificity of political environment with regard to the occupational environment.

\section{Keywords:}

On-the-job training, occupation in politics, sexual division of training, life story, traineeship Journal of Economic Literature: I 29, M 53 
L'exercice des fonctions politiques s'apprend essentiellement sur le tas, c'est-à-dire une fois le mandat électif acquis (Bargel, 2009 ; Demazière, 2009 ; Dulong, Matonti, 2007), mais comment s'opère la socialisation aux rôles politiques ? Les moyens existants pour se former sont peu analysés. Pourtant, ils peuvent aider les élus à exercer leur mandat, en particulier ceux qui sont les moins familiarisés avec le milieu politique. C'est dans ce but que deux lois principales, celle du 3 février 1992 et celle du 27 févier 2002, organisent les droits en matière de formation des élus. Les conseillers municipaux, généraux et régionaux salariés sont autorisés à s'absenter de leur lieu de travail pour suivre des stages relatifs aux fonctions électives, dans la limite d'un congé maximal de dix-huit jours au cours du mandat, quel que soit le nombre de mandats détenus. Ce droit est reconductible après chaque réélection. En outre, depuis 2002, les conseils municipaux ont l'obligation de définir, dans les trois mois suivant leur élection, les orientations et les crédits dédiés à la formation des élus. Cette réforme manifeste une volonté de renforcer la "démocratie de proximité ", comme en atteste l'intitulé de la loi, en facilitant l'accès aux fonctions électives à des groupes sociaux sous-représentés.

Dans ce cadre, la formation revêt un caractère central pour la démocratisation de l'exercice des mandats. Les inégalités d'accès et de suivi peuvent entraver l'investissement dans les pratiques politiques. Par exemple, lors des séances plénières des assemblées, ce sont les élus les plus expérimentés qui parviennent à s'exprimer sans encombre. Il est nécessaire de disposer de solides connaissances sur tous les domaines politiques abordés et d'aptitudes oratoires pour réussir cet exercice qui participe pleinement à la reconnaissance de la capacité des élus à l'être (Navarre, 2015, pp. 262-267). Le suivi de formations thématiques (concernant une question politique précise) ou encore destinées à apprendre les techniques communicationnelles peut faciliter l'adaptation à ces comportements utiles pour ne pas être exclu du débat politique. A contrario, les élus qui en sont les moins familiers, et qui ne peuvent pas se former, risquent de ne pas s'impliquer pleinement dans ces pratiques légitimatrices. Leur engagement restreint et peu reconnu peut les conduire à envisager plus souvent d'abandonner l'exercice du mandat. Ainsi, des inégalités de formation ne favoriseraient pas l'accès de tous aux fonctions électives. En matière de désengagement des activités politiques, le niveau de diplôme et le sexe sont discriminants. Selon notre enquête ( $c f$. Encadré 1), tandis que seulement $9 \%$ des élus ayant un diplôme supérieur à trois années d'études après le baccalauréat envisagent de ne pas renouveler leur mandat, c'est le cas de $20 \%$ des élus disposant d'une qualification inférieure à ce niveau. De même, tandis que seulement $11 \%$ des hommes souhaitent abandonner la politique, c'est le cas de $23 \%$ des femmes. Peut-on expliquer ce désengagement plus fréquent par des difficultés d'accès et de suivi de formations ?

$\mathrm{Ou}$, pour le dire autrement, les lois de 1992 et 2002 favorisent-elles effectivement l'exercice des mandats par les élus, en particulier ceux issus de catégories sociales minoritaires comme les femmes ou les peu diplômés? Plus globalement, comment se formet-on à l'exercice des fonctions politiques ? 


\section{Encadré 1 : Méthodologie d'enquête}

Cette étude est issue d'une thèse de sociologie consacrée aux parcours politiques des femmes élues en Bourgogne. Les données recueillies grâce à l'enquête alliant méthodes quantitatives (questionnaire) et qualitatives (entretiens) abordent la thématique de la formation des élus.

L'enquête par questionnaire, intitulée "Consultation égalité femmes/hommes en politique ", a ciblé les 2000 élus qui siègent au sein d'institutions soumises aux lois sur la parité dans les régions : les conseillers municipaux des communes de plus de 3500 habitants, conseillers généraux, conseillers régionaux, députés et sénateurs.

Les 262 réponses, étudiées grâce au logiciel d'analyse statistique Modalisa, renseignent les parcours politiques des élus, la socialisation politique antérieure ou encore les moyens de formation utilisés.

Malgré le faible effectif de répondants, le corpus reflète la diversité de la population. Il comprend majoritairement des élus municipaux ( $90 \%$ ) et autant de femmes que d'hommes (50\%), comme dans la plupart des institutions politiques étudiées. Les réponses se répartissent équitablement sur le territoire ciblé : les quatre départements qui constituent la Bourgogne sont représentés. De même, les élus qui ont rempli le questionnaire sont majoritairement de gauche, suivant la tendance politique dominante sur le terrain ( 37 des 62 communes ont une majorité de cette tendance ; $28 \%$ des élus consultés revendiquent une appartenance proche du centre droit ou de droite). On observe toutefois une sous-représentation des élus des communes comprenant moins de 5000 habitants ( $17 \%$ des réponses contre $23 \%$ des élus consultés) et, réciproquement, une surreprésentation des communes de plus de 100000 habitants (33\% contre $28 \%$ ). Par ailleurs, $57 \%$ des enquêtés ont déjà exercé au moins deux mandats successifs. Autrement dit, les élus qui ont le plus participé sont ceux qui ont eu le plus d'occasions de recourir aux dispositifs de formation prévus par la loi grâce à l'exercice de mandats depuis plusieurs années, dans des communes disposant de moyens financiers ad hoc.

L'enquête par entretien de type « récit de vie » a été menée principalement auprès de femmes pour approfondir l'analyse de leurs parcours politiques. II s'agit en particulier de conseillères régionales et générales, afin de pallier la sous-représentation de ces élues dans l'enquête par questionnaire. D'une durée moyenne d'une heure trente, ces entretiens abordent les mêmes thèmes que les questionnaires. Les réponses sont toutefois plus développées en raison de la liberté laissée aux enquêtés. 26 femmes et 7 hommes de tendances politiques de droite et de gauche ont participé. L'enquête par entretien comprend des élus membres ou non des exécutifs, novices et expérimentés, ce qui permet de comparer les moyens mobilisés pour se former, selon ces variables. On notera également que même les élus les plus novices ou ayant le moins de responsabilités ont pour caractéristique de cumuler plusieurs mandats électifs. Autrement dit, comme dans l'enquête quantitative, il s'agit d'élus qui ont eu l'occasion de se former en mobilisant les divers moyens à leur disposition.

\section{On distingue schématiquement deux modes d'apprentissage :}

Tout d'abord, la formation peut s'opérer sous la forme de stages dispensés par des organismes publics ou privés : universités, grandes écoles, associations rattachées ou non à une tendance politique, organismes professionnels ou encore cabinets de conseil. Ils doivent être agréés par le Conseil national de la formation des élus locaux (CNFEL), rattaché au 
ministère de l'Intérieur, pour relever des crédits à la formation ${ }^{1}$. Ces stages prennent des formes diverses, d'une durée allant de quelques heures à quelques jours. Ils peuvent être demandés par l'élu individuellement ou par une collectivité locale, pour plusieurs de ses membres. Le recours à ces stages peut être relativement contraignant pour les élus qui éprouvent des difficultés à concilier les sphères d'activité familiale et/ou professionnelle avec leur engagement politique. Par exemple, les élus salariés doivent demander les autorisations d'absence.

Ensuite, on distingue d'autres moyens de formation plus flexibles, notamment la formation informelle et pragmatique, en fonction des besoins (documentation sur un sujet, demande de conseils, etc.). Il s'agit d'étudier l'usage de ces deux modes de formation par les élus locaux, tout en se demandant s'ils favorisent équitablement l'adaptation aux rôles politiques selon le niveau de qualification et le sexe.

L'analyse de la formation des élus locaux révèle des inégalités majeures selon le sexe et le niveau de qualification, comme le montre la première partie à travers la socialisation politique et le recours aux stages. La seconde relativise l'influence de ces derniers sur l'apprentissage du rôle d'élu : il s'opère principalement sur le tas. Les conseillers mobilisent des compétences professionnelles et se renseignent au besoin. Néanmoins, ce mode d'apprentissage, largement partagé, ne permet pas de réduire l'inégale familiarité aux fonctions politiques selon le sexe et le niveau de qualification.

\section{Des élus inégalement formés à l'exercice des mandats}

L'enquête révèle en premier lieu des socialisations inégales à l'exercice des mandats. Celles-ci ne s'atténuent pas en recourant aux stages de formation car ils demeurent peu accessibles, malgré la volonté des élus de se former.

\subsection{Une inégale socialisation aux rôles d'élus}

Afin de mesurer les dispositions des élus à l'exercice de fonctions électives, trois indicateurs ont été retenus. Ils permettent d'appréhender trois types de connaissances nécessaires : les savoirs, les savoir-faire et les savoir-être.

1. Plusieurs critères interviennent pour obtenir l'agrément. Outre le statut juridique, l'identité des dirigeants administratifs et les moyens financiers de l'organisme, le CNFEL se prononce en fonction des moyens matériels (salles de cours, bureaux, équipements pédagogiques), humains (nombre de formateurs et qualifications ; personnel administratif gérant la formation), l'aire géographique d'intervention et, enfin, le programme (modalités de définition des besoins des élus, durée des stages, nom des intervenants, contenus détaillés, effectifs de stagiaires, modalités d'évaluation, coûts financiers). 


\section{Encadré 2 - Lexique : les statuts dans les institutions politiques}

Maire/président : dirigeant de l'assemblée élue.

Adjoint/vice-président : membre de l'exécutif. L'adjoint/vice-président dispose le plus souvent d'une délégation, par exemple, les travaux, les affaires sociales, la culture, les transports, l'école, etc.

Conseiller : élu n'appartenant pas à l'exécutif. II participe seulement aux séances plénières et à certaines commissions.

Le premier indicateur mesure l'aptitude à animer des réunions. Il fait référence à des savoirfaire et des savoir-être comme la capacité à intervenir en public, à organiser et à coordonner le travail, ainsi qu'à se faire écouter et entendre de tous. Cet indicateur teste le leadership des élus.

Le deuxième concerne l'interconnaissance. Il s'intéresse à l'intégration dans les réseaux d'acteurs politiques.

Le troisième se rapporte aux savoirs relatifs aux domaines dont l'élu a la charge. Chacun de ces indicateurs renseigne la situation individuelle avant d'accéder à un premier mandat. Grâce aux réponses des enquêtés, il est possible de construire un indice de socialisation. La note maximale, sur dix points, caractérise les élus qui, avant de l'être, connaissaient la majorité des personnes avec lesquelles ils travaillent dans le cadre de leur mandat, animaient des réunions "souvent" (au moins une fois par mois) et étaient bien informés des enjeux relatifs à leurs délégations. La note minimale caractérise des élus qui connaissaient peu de personnes au sein de l'assemblée d'élection, n'avaient jamais animé de réunions et étaient peu sensibilisés aux enjeux de leurs délégations.

Si cet indice mesure les dispositions des élus au leadership, il est surtout destiné à évaluer la capacité à exercer des responsabilités exécutives. Il s'avère moins adapté pour les "simples » conseillers : par exemple, ils animent peu de réunions dans le cadre de leur mandat. Ces derniers représentent $45 \%$ des répondants. Il faut noter en outre la limite du troisième indicateur mobilisé. Les élus ne cherchent pas nécessairement à obtenir des responsabilités en lien avec leurs connaissances initiales. Cela peut même être contre-indiqué, lorsqu'ils exercent des activités professionnelles susceptibles d'interférer avec les responsabilités électives. L'attribution des délégations s'opère plutôt en fonction des relations entretenues avec les leaders politiques et de l'expérience politique (Navarre, 2013, pp. 474-482). Ainsi, cet indicateur ne reflète pas tant les savoirs de l'élu que les savoir-faire permettant d'obtenir une délégation en lien avec les connaissances initiales. Bien qu'imparfait, l'indice permet d'estimer les dispositions à l'exercice de responsabilités exécutives.

L'indice moyen est de 7,8 sur 10 points. Autrement dit, les élus s'avèrent socialisés aux pratiques politiques avant leur élection. De manière logique au regard des limites précédemment citées, les adjoints le sont plus que les conseillers $(8,1$ contre 7,6$)$. En revanche, 
les inégalités sont notables selon le sexe : les femmes apparaissent moins sensibilisées à l'exercice de responsabilités électives que les hommes (7 points contre 8,6). On ne saurait en conclure qu'elles sont moins socialisées aux pratiques politiques que les hommes, et ce en raison des limites de l'indice qui évalue essentiellement les capacités de leadership. Il n'intègre pas, par exemple, l'aptitude à organiser et à suivre des projets politiques. Or, il s'agit des activités que privilégient plus souvent les femmes, d'autant plus lorsqu'elles sont membres de l'exécutif. Par exemple, $94 \%$ des adjointes observées participent systématiquement aux réunions avec les partenaires de leurs projets politiques contre $76 \%$ des adjoints (Navarre, 2013, pp. 316-319). L'interprétation en termes de socialisation différentielle selon le sexe s'impose dans ce cas.

Enfin, l'indice de socialisation aux rôles d'élu varie en fonction du niveau de qualification, dans une moindre mesure cependant. Les élus diplômés d'un niveau supérieur ou égal au baccalauréat obtiennent un score de 7,9 contre 7,4 pour ceux qui disposent d'une qualification inférieure.

Cet indice révèle donc la familiarité des élus avec les pratiques politiques lorsqu'ils accèdent à un premier mandat. Cependant, les femmes et les élus les moins diplômés présentent des aptitudes plus faibles au leadership. Ces écarts ne sont pas compensés par les formations des organismes agréés.

\subsection{Des stages de formation peu accessibles}

Le recours aux organismes de formation est loin d'être le moyen privilégié par les élus locaux. Rares sont les données sur le sujet. Seulement trois rapports d'activité du CNFEL (Conseil national de la formation des élus locaux) ont été rendus publics. Ils présentent les agréments attribués ou refusés ainsi que les raisons de ces choix entre 2007 et 2011. Cependant, aucune donnée n'existe, à notre connaissance, sur le suivi de ces formations par les élus locaux. Les estimations obtenues à partir de notre enquête montrent qu'à peine un tiers, autant de femmes que d'hommes (31\% et $28 \%$ ), s'est formé par ce moyen pendant l'exercice des mandats. Ce taux est plus faible que dans le milieu professionnel : selon l'enquête "Formation continue " du Centre d'études et de recherches sur les qualifications (Cereq), réalisée en 2010, $45 \%$ des salariés du secteur public et privé ont accès à la formation. Les hommes se forment plus que les femmes ( $47 \%$ contre $42 \%$ ). Ces clivages sont redoublés par l'appartenance socioprofessionnelle : les femmes ouvrières se forment moins que les femmes cadres (Lambert, Marion-Vernoux, 2014 : p. 26 et pp. 40-41). Quelle que soit la catégorie socioprofessionnelle observée, la participation des élus aux stages demeure moindre.

Les difficultés à concilier les différentes sphères d'activité ne permettent pas d'expliquer cette moindre participation des élus aux formations dispensées par les organismes agréés. En effet, la situation professionnelle n'est pas discriminante. Les écarts entre les taux de formation observés parmi les élus actifs et ceux n'exerçant plus d'activité professionnelle 
ne sont pas significatifs. Les modalités spécifiques aux stages, par exemple les thèmes proposés, le déroulé ou encore l'éloignement géographique lorsque l'institution n'en organise pas, semblent donc plus déterminantes dans la participation que la disponibilité des élus.

Le suivi de stages est corrélé au niveau de diplôme. Comme dans le milieu professionnel, ceux qui adoptent le plus ces méthodes d'apprentissage en sont les plus familiers (Dubar, 2004). Plus le niveau de qualification des élus augmente, plus ils sont susceptibles de se former : c'est le cas de $12 \%$ de ceux qui n’ont pas le baccalauréat, contre $39 \%$ des élus diplômés d'un niveau d'étude supérieur.

Le clivage se double d'un effet de genre : ce sont les femmes les plus qualifiées qui suivent le plus de formations ( $41 \%$ des élues diplômées du supérieur contre 36 \% de leurs homologues masculins). En revanche, les femmes les moins diplômées renoncent plus souvent à ces méthodes. Seulement $10 \%$ de celles ayant un niveau inférieur au baccalauréat ont suivi une formation, contre $13 \%$ des hommes. Une moindre socialisation aux apprentissages scolaires détourne des formations, d'autant plus dans le cas des femmes. Ces conclusions prolongent celles relatives au milieu professionnel. Certaines femmes peinent à accéder à la formation continue lorsqu'elles exercent un emploi peu qualifié, à temps partiel, et qu'elles rencontrent des difficultés pour articuler vie professionnelle et vie familiale (Ollagnier, 2010). Les femmes les moins diplômées sont davantage concernées par ce phénomène (Cornet, Laufer, Belghiti-Mahut, 2008). Autrement dit, plus les élus ont un niveau d'études élevé, plus ils ont de chances d'accéder aux stages de formation politique. Ce constat est encore plus valable pour les femmes.

Par ailleurs, la position détenue au sein de l'assemblée influence le suivi de formations. Les membres de l'exécutif, notamment les adjoints au maire, utilisent plus facilement ce mode d'apprentissage que les conseillers municipaux. Tandis que seulement $24 \%$ de ces derniers ont suivi un stage, c'est le cas de $36 \%$ des membres de l'exécutif. Les hommes et les femmes sont aussi souvent dans cette situation. Sachant que tous les élus municipaux disposent des mêmes droits, la fonction d'adjoint semble accroître le besoin de formation. Comme dans le milieu professionnel, ce sont les plus insérés dans les organisations qui en bénéficient le plus.

La prise en compte de l'ancienneté élective confirme cette interprétation. Les novices ${ }^{2}$ sont ceux qui se sont le moins formés alors que, paradoxalement, c'est lors des premières années d'exercice d'un mandat que la connaissance et la maîtrise des fonctions d'élu sont les plus faibles. Plus la longévité politique augmente, plus les chances de suivre des stages progressent. Ceux qui sont élus depuis 2001 en ont bénéficié davantage que ceux élus à partir de 2008 (39 \% contre 28 \%). Les stages permettent de perfectionner des compé-

2. Nous désignons par le terme de "novice ", les élus qui n’ont pas encore achevé un premier mandat. La plupart des répondants étant des conseillers municipaux, il s’agit principalement de conseillers qui débutent leur deuxième année d'élection au moment de l'enquête (2010). Les élus expérimentés ont achevé l'exercice d'au moins un mandat. 
tences politiques précises (budget de la collectivité, communication avec les médias, organisation de la politique culturelle, etc.), mais moins souvent d'acquérir des savoirs de base et des savoirs pragmatiques sur les fonctions d'élu, particulièrement utiles aux novices (par exemple, comment gérer son temps face aux multiples activités ?).

Sans présumer de son efficacité, on peut conclure que la loi de 2002 qui encadre la « formation en début et en cours de mandat " la favorise essentiellement en cours de mandat. En outre, elle tend à reproduire les mêmes inégalités que dans le monde du travail, même si les contextes sont différents : ce sont les individus les plus intégrés au sein des organisations (adjoints, élus expérimentés et diplômés, hommes) qui profitent davantage de ces dispositifs. Ce sont également les plus familiers des pratiques politiques, ce qui redouble les difficultés que peuvent rencontrer les élus novices, peu diplômés et les femmes pour s'adapter à leurs rôles publics. Ces clivages se prolongent à travers les besoins de formation non-satisfaits.

\subsection{Des besoins de formation non satisfaits, surtout chez les femmes}

Plus des deux tiers des élus auraient souhaité suivre davantage de formations, en particulier les femmes et ceux disposant d'un niveau d'étude modeste. La position au sein de l'assemblée (membre ou non de l'exécutif) et l'expérience politique ne sont pas discriminantes dans ce $\operatorname{cas}^{3}$ : les élus novices et les "simples » conseillers éprouvent aussi souvent le besoin de suivre des stages que les autres. La non-satisfaction des souhaits de formation est nettement plus prononcée dans le milieu politique que dans le milieur professionnel, où elle concerne seulement deux salariés sur dix (Lambert, Marion-Vernoux, 2014, p. 74).

Le niveau de diplôme intervient dans ce phénomène. Ce sont en particulier les élus disposant d'une qualification moyenne, voire faible - mais qui n'en sont pas pour autant totalement dépourvus - qui ont davantage besoin de formation : $85 \%$ des diplômés d'un niveau baccalauréat auraient voulu participer plus souvent à des stages. Au contraire, les moins diplômés se montrent moins sensibles à ces dispositifs, même s'ils restent majoritairement convaincus de ne pas assez y recourir. $59 \%$ d'entre eux déclarent ne pas s'être formés autant qu'ils l'auraient souhaité. Cette part est moins élevée que chez les diplômés du supérieur (69\%), mais elle demeure inférieure à celle des diplômés du baccalauréat.

En résumé, la plupart des enquêtés souhaitent se former davantage, en particulier ceux qui disposent d'un niveau d'étude général peu élevé, comme le baccalauréat.

On peut expliquer ces constats par la moindre sensibilité des élus peu ou pas diplômés aux formations théoriques et formelles. Pour ces derniers, des apprentissages pratiques s'avèreront sans doute être des méthodes plus familières. Elles seront donc privilégiées. Quant aux élus les plus diplômés, on peut supposer que leur qualification fournit quelques

3. Les tests du Khi-deux ne sont pas significatifs au seuil de $5 \%$. 
connaissances utiles en politique, par exemple, en termes de management ou de leadership. Dès lors, ils peuvent moins ressentir le besoin de se former.

Le sexe constitue une seconde variable discriminante. Les souhaits de formation des femmes sont plus manifestes que ceux des hommes : $77 \%$ d'entre elles déclarent qu'elles auraient aimé suivre davantage de stages, tandis que ce n'est le cas que de $62 \%$ des hommes.

Tableau 1 : Souhaits de formation selon le sexe (en \%, 2010)

\begin{tabular}{|l|c|c|c|}
\hline & Hommes & Femmes & Ensemble \\
\hline Aurait voulu suivre plus de formations & 62 & 77 & 69 \\
\hline N'aurait pas voulu en suivre davantage & 38 & 24 & 31 \\
\hline Total & $100(113)$ & $101(115)$ & $100(228)$ \\
\hline
\end{tabular}

Test du Khi-deux significatif $(p=0.024)$

Source : Consultation égalité femmes/hommes en politique - Bourgogne.

Les obstacles rencontrés par les femmes pour développer leurs compétences dans le domaine professionnel sont nombreux. Le manque de temps en raison des impératifs de conciliation avec la vie familiale qui pèsent plus souvent sur elles (Fournier, 2009), l'absence d'offre à cause de l'exercice d'activités dans des secteurs et sous des formes qui ne favorisent pas la l'apprentissage en continue (emplois peu qualifiés, précaires et à temps partiel dans le domaine des services non-marchands), l'éloignement géographique du lieu de formation ou encore le manque d'information constituent autant de facteurs discriminants (Brocard, De Cidrac, Fournier, 2002). Certains de ces facteurs opèrent aussi dans le champ politique. Les femmes élues déclarent souvent ne pas avoir le temps d'aller en stage. L'éloignement géographique des organismes formateurs ${ }^{4}$ contribue sans doute à ce phénomène en engendrant des absences du foyer que les personnes ayant des responsabilités familiales, souvent des femmes, ne peuvent pas toujours se permettre. Enfin, les formations se centrent sur les fonctions et responsabilités politiques les plus prestigieuses (maires, présidents d'assemblées, membres d'exécutifs en charge de compétences régaliennes comme les finances, l'aménagement du territoire ou l'économie) qui concernent moins souvent les femmes que les hommes. Elles occupent en effet plus souvent des fonctions politiques subalternes, en charge de compétences telles que les affaires sociales ou encore scolaires (Navarre, 2013, pp. 240-270).

Lorsqu'ils accèdent à un premier mandat, les élus s'avèrent relativement socialisés à l'exercice des fonctions électives, comme en atteste l'indice permettant de mesurer la familiarité avec quelques pratiques politiques. Cependant, ils le sont inégalement. Les femmes et les moins diplômés présentent une moindre sensibilisation. Les stages de formation ne permettent pas de combler ces lacunes car seule une minorité y recourt. Ils sont diverse-

4. On compte 196 organismes accrédités en 2013 dont un quart à Paris même. Source : Conseil national de la formation des élus locaux. 
ment employés selon le niveau de qualification ou encore le sexe. Si les moins diplômés ne souhaitent pas nécessairement les utiliser, les femmes en revanche manifestent la volonté d'y recourir plus fréquemment. Dans leur cas, l'accès à la formation est empêché par des problèmes de conciliation entre vie privée, professionnelle et politique, ainsi que par une offre de formation ne correspondant pas nécessairement à leurs responsabilités politiques. D'autres modes d'apprentissage sont privilégiés.

\section{La socialisation aux rôles politiques: un apprentissage sur le tas}

Ladaptation aux rôles politiques relève principalement de moyens informels, autrement dit, d'un apprentissage sur le tas. Il s'agit de l'une des caractéristiques essentielles des fonctions électives locales. Notre enquête permet de décrire précisément ce processus : elle révèle que les élus mobilisent principalement les connaissances acquises hors du milieu politique, sollicitent des conseils ponctuels et utilisent de la documentation.

\subsection{La mobilisation des savoirs professionnels antérieurs}

L'une des hypothèses de notre enquête envisageait le militantisme comme une ressource pour les élus. L'exercice d'activité, voire de responsabilités militantes, fournirait des connaissances et des pratiques utiles à tous les mandats politiques. Or, les savoirs professionnels s'avèrent les plus utiles. Ils peuvent garantir, tout d'abord, une connaissance du fonctionnement du milieu politique. Par exemple, une adjointe au maire et conseillère générale, élue depuis deux ans au moment de l'enquête, par ailleurs directrice adjointe d'un bailleur social, explique que son emploi l'a confrontée aux rouages administratifs des institutions politiques, en particulier des services sociaux du conseil général, ce qui lui fut d'une utilité certaine, étant chargée des affaires sociales :

"Le fait de travailler dans le logement social m’a aidée. Je travaille pour un bailleur social qui est en contact avec le conseil général, organisme de tutelle. Je connaissais déjà le fonctionnement et les interlocuteurs, même si je suis élue dans un autre conseil général. " Conseillère générale (opposition) et membre d'un exécutif municipal depuis 2008, quadragénaire, directrice adjointe.

La profession peut ensuite garantir des savoir-faire transposables à l'exercice des mandats, notamment pour le travail des dossiers. La formation universitaire a été présentée par un tiers des élus du corpus d'entretiens comme favorisant la prise en charge des projets politiques. C'est le cas par exemple d'un adjoint au maire en poste depuis une dizaine d'années, professeur des universités. Il explique que l'esprit de synthèse et l'art de la présentation appris grâce à sa profession lui ont permis de se familiariser efficacement à une délégation jusque-là méconnue : l'urbanisme. 
"Je n'y connaissais rien! Ma formation universitaire m'a permis de m'en sortir en travaillant beaucoup, en potassant les dossiers et en mettant de l'ordre dans tout ça... Je me suis enfermé pendant des jours et j'ai lu. Les services avaient préparé un dossier volumineux. Je l'ai lu en entier, en faisant une synthèse comme un universitaire sait faire. " Membre d'un exécutif municipal depuis 2001, quinquagénaire, professeur des universités.

Ces connaissances citées pour leur utilité en politique sont plutôt caractéristiques des professions intermédiaires, voire des cadres et des professions intellectuelles supérieures. Les élus n'appartenant pas à ces groupes sociaux ne peuvent pas s'en prévaloir. Ils représentent pourtant une part non négligeable (46\% parmi l'ensemble des conseils municipaux des villes de plus de 3500 habitants en France) ${ }^{5}$. Par ailleurs, la reproduction de la division du travail selon le sexe dans le champ politique, génère des inégalités en matière de reconversion des aptitudes professionnelles. Les femmes sont moins souvent cadres et issues des professions intellectuelles supérieures (Navarre, 2013, pp. 291-303).

Par conséquent, elles ne peuvent remobiliser les compétences caractéristiques de ces groupes professionnels (capacités de synthèse, de traitement d'un grand nombre d'informations ou encore de restitution). En revanche, certaines évoquent l'utilité des aptitudes relationnelles et communicationnelles acquises grâce à l'exercice de professions majoritairement féminines. Par exemple, une enseignante explique que ce métier lui fournit des savoir-faire utiles en politique, comme la capacité à "travailler en groupe " et à "mener les discussions ". Plus caractéristique des professions intermédiaires, groupe le plus représenté chez les femmes, une infirmière précise que l'exercice de son métier lui a appris à développer une forme de "bienveillance " utile dans sa fonction de conseillère générale, d'autant plus qu'elle considère que son rôle d'élu consiste avant tout à entendre et relayer les attentes des administrés auprès des leaders politiques :

"Indéniablement, mon métier d'infirmière m'a aidée! Quand les gens arrivent pour se faire opérer, par exemple à coeur ouvert, ils ont le trouillomètre à zéro. En quelques minutes, il faut sentir la personne et deviner les mots qu'elle a besoin d'entendre ou les gestes qui l'apaiseraient. C'est un travail d'acceptation de l'autre tel qu'il est. Il oblige à être toujours dans la bienveillance. J'ai la prétention de croire que c'est quelque chose qui m'est resté dans ma façon d'être en politique.» Conseillère régionale (opposition) et générale (majorité) depuis 2008, quadragénaire, infirmière.

La profession fournit alors des savoir-être utiles en politique, notamment dans le cadre de la relation avec les administrés.

Concernant l'utilité des savoirs militants, ce thème n'a été abordé qu'avec les élus enquêtés par entretien. Seulement trois d'entre eux, toutes des femmes, ont réutilisé les pratiques acquises dans les organisations partisanes, syndicales et/ou associatives. Deux ont pour point commun d'avoir milité dans le milieu de l'enseignement, domaine par ailleurs plutôt

5. Source : ministère de l'Intérieur, 2008. 
féminisé. L'une est syndiquée dans un établissement scolaire ; l'autre s'est engagée dans une organisation de parents d'élèves. La dernière travaille dans une entreprise privée. Le militantisme fournit des savoirs tels que la connaissance du fonctionnement des institutions politiques et des savoir-être, comme l'attitude à adopter face aux revendications des administrés. Ils rejoignent les compétences acquises par la profession. Par exemple, un membre de l'exécutif du conseil régional, élue depuis six ans, mentionne l'utilité de son engagement syndical pour saisir les inquiétudes liées au changement de statut du personnel TOS (techniciens, ouvriers et de service) et ainsi, désamorcer les hostilités potentielles à cette mesure.

"Mon mandat syndical m'a aidée : par exemple, quand on a fait le transfert des TOS. Je fais partie d'un Comité technique paritaire. J'avais senti l'inquiétude du personnel de l'État transféré à la région. J'ai porté un discours rassurant. Le président du conseil régional voulait vraiment faire un rattrapage salarial. On voulait quils soient traités de la même manière que le personnel administratif au niveau de la grille des salaires. Grâce à mon expérience de représentante syndicale, j'ai compris leurs inquiétudes. " (Membre de l'exécutif du conseil régional, élue depuis 2004, quadragénaire, enseignante).

La sous-représentation des hommes parmi le corpus d'enquêtés par entretiens explique certainement la faible utilité déclarée du militantisme. En effet, d'une part, ces pratiques sont plus fréquentes et diversifiées chez les hommes que chez les femmes. Elles sont multiformes (engagement partisan, syndical, associatif). D'autre part, elles se caractérisent plus souvent par l'exercice de responsabilités exécutives comme la direction d'organisations militantes (Navarre, 2013, pp. 276-291). Il conviendrait donc de vérifier systématiquement l'usage du militantisme dans l'exercice des mandats politiques pour conclure à son utilité.

L'expérience professionnelle demeure la principale ressource en politique. Or, ce ne sont pas n'importe quelles connaissances qui s'avèrent utiles. Il s'agit principalement des connaissances caractéristiques des milieux socioprofessionnels intermédiaires, voire supérieurs. Des inégalités transparaissent alors, au détriment des employés et des ouvriers, voire des sans-emplois. Par ailleurs, l'inégale répartition des professions et de l'expérience militante selon le sexe amène les femmes à ne pas réutiliser le même type d'aptitudes que les hommes. Tandis que ces derniers semblent pouvoir s'adapter plus facilement à une diversité de délégations grâce à leurs capacités de synthèse, les femmes le font également, mais grâce à leurs aptitudes relationnelles. Elles leur permettent, par exemple, de désamorcer les conflits. Cependant, la formation sur le tas ne se résume pas à la transposition d'aptitudes initiales. Le milieu politique contribue lui-aussi à une formation informelle et pragmatique. 


\subsection{L'usage de modalités informelles et pragmatiques}

Les élus intègrent de nouveaux comportements sur le tas, en fonction des situations dans lesquelles ils sont impliqués. Afin de saisir ce mode informel d'apprentissage, nous avons mobilisé deux indicateurs principaux : le recours à de la documentation sur le mandat d'élu et la demande de conseils. Les élus utilisent davantage ces modalités informelles et pragmatiques que les stages de formation : $82 \%$ d'entre eux déclarent avoir eu recours à de la documentation et $98 \%$ demandent conseil à leur entourage.

Le niveau de qualification et le sexe ne sont pas discriminants dans ces deux cas : les écarts enregistrés ne sont pas significatifs au seuil de $5 \%$. D’autres variables interviennent, notamment dans l'usage de la documentation. Il s'agit en particulier de l'expérience politique et de la position détenue au sein de l'assemblée. Dans le premier cas, les élus les plus novices déclarent moins souvent que les expérimentés se documenter sur leur rôle (74\% contre $91 \%$ ). Ces données confirment le fait que la formation intervient en cours de mandat et non pas dès le début. Dans le second cas, ce sont les conseillers municipaux d'opposition qui se documentent le plus (94\%), suivis des membres de l'exécutif (86\%) et, enfin, des conseillers de la majorité (65\%). La position détenue au sein de l'assemblée est donc déterminante. Ce sont surtout les élus les plus en marge ou, au contraire, les plus intégrés au sein de l'institution politique qui se documentent.

La situation des conseillers d'opposition peut s'expliquer par leurs plus rares occasions de se former grâce à l'apprentissage sur le tas, au contact des autres élus. En entretien, ceux qui ont siégé dans des groupes minoritaires évoquent leur position marginalisée et stigmatisante. La situation d'opposant encourage la majorité et les entourages politiques tels que les services techniques par exemple, à ne communiquer que les informations strictement nécessaires au sujet des mesures adoptées et du fonctionnement institutionnel. Par conséquent, le besoin de documentation est plus important afin d'adopter les comportements adéquats. Les membres de l'exécutif, pour leur part, doivent s'informer en raison des responsabilités qui leur sont confiées. Enfin, les conseillers de la majorité se documentent un peu moins car ils ont un rôle secondaire dans le jeu municipal (pas de fonction de leadership, ni d'opposition). Comme l'acquisition informelle des pratiques professionnelles, qui peut constituer une alternative aux stages de formation (Carré, Charbonnier, 2003), les rôles d'élu s'apprennent principalement à travers les échanges de conseils quotidiens avec les autres acteurs et l'autoformation, grâce à la documentation. Ces modes d'apprentissage semblent plus faciles d'accès que les stages puisque le sexe et le niveau de qualification ne sont pas discriminants. Ce sont plutôt des variables politiques, comme l'expérience et la position au sein de l'assemblée, qui le sont. Développer ce type de formation sur le tas pourrait permettre de pallier les inégalités en matière de socialisation politique initiale et de suivi des stages. 


\section{Conclusion}

Notre enquête révèle la spécificité des processus d'apprentissage mobilisés par les représentants politiques pour s'adapter à leurs fonctions. La formation formelle, à travers des stages, est davantage délaissée que dans le milieu professionnel. Les élus n’utilisent pas ou peu les droits à la formation dont ils bénéficient. Les deux tiers des conseillers rencontrés n'ont jamais suivi de stage, alors qu'ils siègent dans les institutions politiques susceptibles d'attribuer le plus de moyens aux élus dans ce but (conseils municipaux des communes comprenant un grand nombre d'habitants, conseillers généraux et régionaux). Par ailleurs, le recours aux stages est clivé selon le sexe et le niveau de qualification. Les élus les plus diplômés et les hommes y accèdent davantage. Cependant, ces clivages sont renforcés par d'autres, propres au milieu politique : ce sont principalement les élus les plus intégrés au sein des institutions, notamment les membres de l'exécutif et les conseillers ayant déjà exercé de précédents mandats, qui y accèdent. Autrement dit, les dispositifs de formation bénéficient essentiellement aux élus déjà insérés dans le champ politique. Ils renforcent leur motivation à l'engagement, tandis que ceux qui n'y accèdent pas, et qui sont également les moins familiers des pratiques politiques, peuvent rencontrer davantage de difficultés pour s'impliquer dans leurs responsabilités élective, voire être découragés au point de penser à abandonner. Le champ politique reproduit les inégalités sociales en matière de formation, tout en les renforçant selon la position détenue au sein de l'assemblée et l'expérience élective.

Plus globalement, l'ensemble des élus mobilise davantage un apprentissage sur le tas. On peut distinguer, en particulier, la reconversion d'aptitudes antérieures qui, comme dans le cas de la formation continue, profite essentiellement aux élus les plus diplômés, notamment ceux qui exercent des professions intellectuelles leur conférant des aptitudes de synthèse et des capacités à traiter un grand nombre d'informations en même temps. En revanche, le sexe est un peu moins discriminant dans ce cas, puisque les femmes peuvent aussi mobiliser en politique des savoir-être professionnels tels que l'écoute ou l'empathie, plutôt caractéristiques des métiers de service.

Enfin, les modalités informelles de formation, telles que la documentation ou la demande de conseils, sont utilisées par la très grande majorité des élus, de manière relativement identique selon le sexe et le niveau de qualification, ce qui ne permet pas à ceux qui sont les moins familiers des pratiques politiques de compenser leurs lacunes par un recours plus fréquent à ces modes d'apprentissage.

Ainsi, les dispositifs de formation sont inégalement utilisés par les élus. Des solutions existent pour réduire ces clivages. Notre enquête montre que ces derniers se centrent principalement sur le suivi des stages, tandis que l'apprentissage sur le tas est accessible au plus grand nombre, sans permettre pour autant à ceux qui sont les moins familiers de la politique de combler leurs lacunes en y recourant davantage. Dès lors, l'une des solutions pour favoriser la formation de tous les élus consisterait à développer les réseaux d'entraide. Des systèmes de parrainage pourraient être instaurés entre les élus novices et les élus expérimentés, afin de favoriser l'intégration des nouveaux conseillers grâce à l'échange d'informa- 
tions. Il s'agirait ainsi de " coacher " les nouveaux entrants. Quant aux stages, ils s'avèrent être des modes de formation davantage adaptés aux hommes et aux personnes fortement diplômées. Ils sont peu attractifs pour les autres. Il conviendrait de revoir leur forme pour qu'ils suscitent l'intérêt de tous les élus. Il s'agit là d'un enjeu essentiel afin que la politique ne soit pas exclusivement réservée à une élite sociale.

\section{Bibliographie}

Achin C. (2005), "Le mystère de la chambre basse ", Comparaison des processus d'entrée des femmes au Parlement France-Allemagne 1945-2000, Dalloz, Paris.

Bargel L. (2009), Jeunes socialistes, jeunes UMP. Lieux et processus de socialisation politique, Dalloz, Paris.

Brocard C., De Cidrac G., Fournier C. (2002), L'accès des femmes à la formation continue et ses effets sur leurs carrières, La Documentation française, Paris.

Carré P., Charbonnier O. (dir.) (2003), Les apprentissages professionnels informels, L'Harmattan, Paris.

Cornet A., Laufer J., Belghiti-Mahut S. (coord.) (2008), GRH et genre : les défis de l'égalité hommes-femmes, Vuibert, Paris.

Demazière D. (2009), « Les élus locaux débutants. Découverte du travail politique et production des professionnels » in Demazière D., Gadéa C. (dir.), Sociologie des groupes professionnels. Acquis récents et nouveaux défis, La Découverte, Paris, pp. 197-208.

Dubar C. (2004), La formation professionnelle continue, La Découverte, Paris.

Dulong D., Matonti F. (2007), "Comment devenir un(e) professionnel(le) de la politique ? L'apprentissage des rôles au conseil régional d'Île-de-France ", Sociétés et représentations, $\mathrm{n}^{\circ} 24$, pp. 251-267.

Fournier C. (2009), "Concilier vie familiale et formation continue, une affaire de femmes », Bref-Céreq, n ${ }^{\circ} 262$.

Lambert M., Marion-Vernoux I. (2014), Quand la formation continue. Repères sur les pratiques de formation des employeurs et des salariés. État des lieux à l'aube de la réforme de 2014, Cereq, Marseille.

Navarre M. (2015, à paraître), « Prendre la parole en séance plénière : des inégalités sexuées persistantes ", Travail, genre et sociétés, $\mathrm{n}^{\circ} 33$.

Navarre M. (2013), Des carrières politiques sous contraintes de genre. Le cas des élues en Bourgogne, Thèse pour le doctorat de sociologie, université de Bourgogne, 665 p.

Ollagnier E. (2010), "La question du genre en formation des adultes ", Savoirs, n 22, pp. 9-52. 


\section{L'ORIENTATION SCOLAIRE ET PROFESSIONNELLE}

Revue de l'Institut national d'étude du travail et d'orientation professionnelle

Numéro 43/3 (septembre 2014)

Émilie Vayre, Anne-Marie Vonthron \& Jean Vannereau

Effets des motifs d'entrée, des croyances d'efficacité personnelle et de l'engagement en formation sur le maintien, l'échec et la réussite des étudiants en situation de e-learning

Fabienne Rousset \& Patricia Rossi-Neves

Entrer à l'Institut universitaire de technologie ou à l'université : un choix d'orientation révélateur du rapport au savoir

\section{Michel Tondellier}

Les familles à l'épreuve des ruptures scolaires

Geneviève Fournier, Hélène Zimmermann, Christine Gauthier \& Jonas Masdonati

L'expérience du chômage chez les travailleurs seniors : de la crise identitaire à la réflexion sur l'avenir

Abonnement 2015 (4 numéros par an)

France : 112 Euros, Étranger : 118 Euros, Vente au numéro : 45 Euros

OSP - Service abonnement

41 rue Gay-Lussac - 75005 Paris

0144107833 - inetop-osp@cnam.fr 